\title{
Benefits of Measuring the Transcutaneous Bilirubin in Neonates: Its Role in Preserving the Quality of Examination, Manipulation and Cost Effectiveness
}

\author{
Elona Gjylbegaj ${ }^{1}{ }^{2 *}$, Niketa Koliçi ${ }^{2}$, Rustem Celami ${ }^{3}$ \\ 1 Resident in Pediatrics at UMT, Mother Teresa University Hospital Center, Tirana \\ 2 Neonatologist, Head of Neonatology Department, American Hospital Tirana 3 \& lecturer at U. Elbasan \\ 3 Obstetrician- Gynecologist, Obstetrics and Gynecology Department - American Hospital Tirana 3, Tirana, \\ Albania \\ *Corresponding author Elona Gjylbegaj:elonagjylbegu@gmail.com
}

\begin{abstract}
Introduction: Hyperbilirubinemia is one of the most common diagnoses of neonatal period affecting about $60 \%$ of babies born around term ${ }^{1,8}$. The clinical evaluation of jaundice only through inspection is insufficient. The most accurate way of assessing it is measuring the value of venous bilirubin (VB), which is an invasive method. A simpler way is to measure transcutaneous bilirubin (TB), a spectrometric method that evaluates the bilirubin based on skin color ${ }^{1,2}$.

Objectives: This study aims to test the effectiveness of the TB measurements in newborns, the impact on the frequency of manipulations and the cost-effectiveness of this procedure in the neonatology discipline in our country.

Materials and methods: This retrospective study was conducted with a sample of neonatal population of a total of 1044 individuals, at the American Hospital 3, Tirana. With regard to the patients data collected from MarchAugust 2017, 505 neonates were part of the study, 22 of which were excluded when the decision to take VB or from them was left at the doctor's judgment. With respect to the patients data collected from March-August 2018, there were 539 neonates part of our study, 8 of which were excluded when the decision to take VB or not depended on the TB values.

The exclusions criteria were: neonatal weight $<2000 \mathrm{gr}$, gestational age $<34$ weeks, other pathologies that required NICU care, blood sample taken after the 14th day of life, and when data were incomplete. The quantitative and qualitative data of the total sample in the two years of the respective study were evaluated and analyzed according to the relevant protocols for the scientific study, using SSPI 2019.

Results: It turned out from the evaluated and analyzed data of the population of the study, from 1044 subjects neonates, out of whom, 505 belonged to 2017 and 539 belonged to 2018, that in the period March - August 2017, there were 483 neonates included in the study, of whom, 249 males and 234 females, 18 less than 2500 gr, 77 less than 3000 gr, 283 between 3000-4000 gr, 105 over 4000 gr. In terms of gestational age, 18 were less 37 weeks and 465 over 37 weeks. As regards the 2018 period, the study included 531 neonates, of whom, 272 males and 259 females, 16 less than $2500 \mathrm{gr}, 95$ less than $3000 \mathrm{gr}, 381$ were among 3000 - 4000 gr, 39 were over 4000 gr; 28 of them were less than 37 weeks, 513 were over 37 weeks. It was observed also that during 2018, the neonatal manipulations have been decreased from $39.8 \%$ to $24.2 \%$, consequently, it corresponds integrally to reduced costs.

Conclusions: At the end of the study it was found that the use of transcutaneous bilirubin significantly reduces the number of manipulations in infants.
\end{abstract}

Keywords: Bilirubin, jaundice, neonate, transcutaneous

DOI: $10.7176 /$ ALST/92-03

Publication date: February $28^{\text {th }} 2022$

\section{Introduction}

Hyperbilirubinemia is a common finding in newborns. Clinically it is presented with jaundice on the skin and sclera, which comes as a result of increased production of bilirubin. The physiological jaundice is very common in $60 \%$ of babies born around term ${ }^{1,8}$, but only $6 \%$ of newborns develop pathological hyperbilirubinemia, which may become neurotoxic and may lead to permanent neurological sequelae, and even death ${ }^{1,2}$. Therefore hyperbilirubinemia requires careful monitoring.

Neonatal jaundice is typically noticed first around the second day of life on the baby's face, progressing to trunk and extremities, reaching its peak by the 5th day and it is usually disappears by the end of the second week, in the opposite direction ${ }^{1,4}$. The increase of bilirubin levels in newborns comes as a result of the imbalance between increased production and its slower elimination.

Increased bilirubin excretion results from the shorter lifespan of the fetal erythrocyte as well as the increased number of erythrocytes in neonates ${ }^{1}$. 
The slower elimination in neonates is due to the low concentration of ligand-binding protein in the hepatocyte, and the low activity of glucuronyl transferase, the responsible enzyme for bilirubin conjugation ${ }^{4}$.

Although a transient physiological phenomenon, neonatal jaundice may be more dangerous in a defined category of newborns, to whom we need to take extra care in assessing jaundice.

Among the risk factors for hyperbilirubinemia should be mentioned: blood group incompatibility (Rh, ABO, etc.); non-immune hemolytic disorders, such as spherocytosis, glucose-6 dehydrogenase deficiency; certain factors found in the breast milk of some mothers that affect the control of enterohepatic bilirubin circulation; Gilbert's children syndrome associated with genetic disorders; prematurity and birth weight; congenital infections; inadequate nutrition; genetic factors associated with hyperbilirubinemia, history of children and other $\operatorname{aspects}^{2,4,8}$.

The assessment of jaundice and risk for hyperbilirubinemia is based on life hours and risk factors. The gold standard for assessing jaundice is laboratory examination and measurement of venous bilirubin. The VB remains the safest way for the right evaluation of hyperbilirubinemia and the decision for phototherapy is made only on the basis of VB values. However, one should be aware that such a way is invasive, painful for babies, in some cases, as in premature infants frequent tests can become a cause for anemia of the newborn. It requires qualified nursing staff to take samples as well as to analyze them, it takes time to get the result, and it also has a considerable cost considering that it is a very frequent examination in the neonatal period.

An alternative way to assess jaundice is to measure TB, which is a quick way - the result is obtained directly, it is a non-invasive method, very easy to use and at a relatively lower cost ${ }^{1.2 .8 .9}$.

The transcutaneous bilirubin measurement is a spectrometric method that basically measures the intensity of the wavelength that returns after directing light into the baby's skin. It is based on optical spectroscopy, which correlates the amount of light absorption by bilirubin with the concentration of bilirubin in the skin ${ }^{8}$.

This technology was first introduced in 1980 by Yamanouchi and since then numerous studies have been conducted to compare between the two methods, can TB measurement replace VB, and what are the advantages and limitations of them. The main components that affect TB measurements in the reflection of the light spectrum are: melanin, dermal maturity, hemoglobin, and bilirubin. The most optimal is the use of multiwavelength bilirubin meters.

Phototherapy, venous immunoglobulin (IVIG) and exsanguinate transfusion are therapeutic options in children with pathological hyperbilirubinemia 2, 4 . Despite the impact of many medications on bilirubin metabolism, studies have shown that the use of drugs does not sufficiently affect the unconjugated bilirubin of neonates ${ }^{4}$. The primary treatment is phototherapy. The decision whether a child should undergo phototherapy is always based on VB values. It should also be known that in a child who has undergone phototherapy, BT effectiveness decreases. The use of IVIG or exsanguinate transfusion is for high risk babies and very toxic bilirubin values, conditions that are also associated with other pathologies.

\section{Materials and methods:}

In this study we have tried to show the effectiveness of using TB measurement compared to VB measurement. It is a retrospective study, extended respectively in the same periods of time March - August 2017 and 2018 . It compares the data obtained from the patient cards and the hospital laboratory.

The study includes all births registered at the American Hospital 3 Tirana from March - to August 2017. In this period, the main way to evaluate the neonatal jaundice was made only clinically, the doctor, based on the examination, decided whether to take the VB. The study evaluates the number of babies who needed VB, how many blood tests for babies have been taken, the number of babies who needed phototherapy, laboratory cost for each baby, etc. Specific data were categorized by gender, weight, gestational age and nationality (Albanian or foreign).

The same data were collected for babies born in the same hospital, in the same period, March-August of a year later, 2018 - when in this clinic began the use of TB measurement. The decision to take VB depended on the preliminary value of TB.

Considering the limitations of TB measurement, this study includes only those babies born over 34 weeks of gestational age and weight over 2000 gr. Babies diagnosed with serious pathologies other from hyperbilirubinemia are also excluded so as not to artificially increase the number of VB taken. Another exception were babies for whom the data were incomplete.

According to the protocol established in the American Hospital 3 neonatology clinic, the babies from mothers with blood group 0 (I) and Rh (-) neg. should be evaluated for bilirubin levels from umbilical cord blood. It were excluded all these data taken from umbilical cord blood. It was considered only the bilirubin values taken in the first 2 weeks of life, no later.

\section{Equipment and techniques used:}

To measure the TB we used a KJ-8000 bilirubinometer (fig.1). The TB is measured in the baby's sternum and 
for values above $13 \mathrm{mg} / \mathrm{dl}$ it is required the taking of $\mathrm{VB}$.

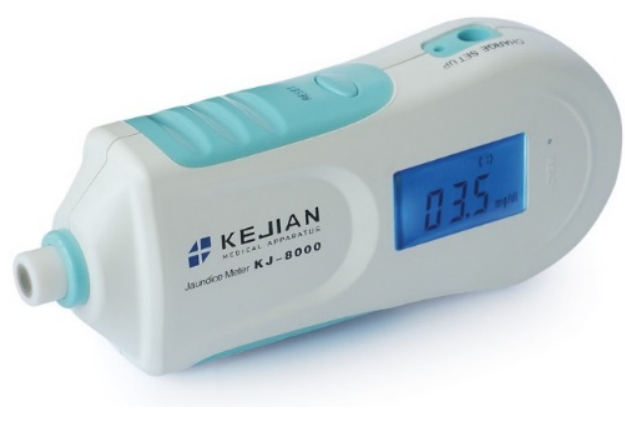

Fig. 1 -Bilirubinomether used in American Hospital

\section{Bhutani curve: phototherapy}

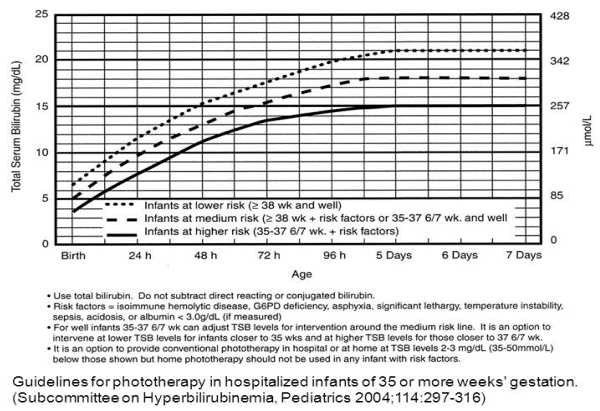

Fig . 2-Bhutani's curves

In all cases the decision for the phototherapy was based on VB measurement. VB values were assessed according to Bhutan curves, and the decision for phototherapy was made according to them (fig.2).

\section{Results}

After processing the data, summarized in Table 1, it is concluded that using TB decreases significantly the number of babies who need blood tests, from $39.8 \%$ to $24.2 \%$ and consequently the costs. If in 2017 for each baby we would add a 0.66 of the value of VB, in 2018 this cost would be reduced to 0.39 of its value.

It should be noted that each method used does not influence bilirubin taken for each neonate, it is the same 1.66 1.61, which confirms the importance of clinical judgment. Also, the number of babies in phototherapy is comparable to $5.1 \%$ and $4.3 \%$, less than $6.5 \%$ of the incidence, without being affected by the method chosen to be used.

It has been concluded that the female/male ratio has no effect on increased bilirubin values.

The results of this study suggest that the use of TB measurement significantly reduces the number of blood tests in babies and it is cost effective as well. According to our study its application is fully recommended, despite its limitations.

\section{Results from 2017}

Between March and August 2017, there were 505 babies born at American Hospital 3, of which 483 babies included in the study. 22 babies were excluded for the following reasons: 3 due to weight $<2000 \mathrm{gr}, 4$ for gestational age $<34$ weeks, 5 for associated pathologies for which they needed a long stay in NICU, 5 babies whose bilirubin was taken after the $14^{\text {th }}$ day of life and 5 babies for incomplete data.

The following table (tab.1) shows the data grouped by gender, weight, gestational age and nationality.

Tab. 1

\begin{tabular}{|lll|}
\hline Total cases of the study & $\mathbf{4 8 3}$ \\
\cline { 2 - 3 } Gender & $\mathrm{M}$ & 249 \\
\hline Weight & $\mathrm{F}$ & 234 \\
& $<2500 \mathrm{gr}$ & 18 \\
\cline { 2 - 3 } & $<3000 \mathrm{gr}$ & 77 \\
\cline { 2 - 3 } & $3000-4000 \mathrm{gr}$ & 283 \\
\cline { 2 - 3 } & $4000 \mathrm{gr}$ & 105 \\
\hline Gestational age & $<37$ weeks & 18 \\
\cline { 2 - 3 } & $>37$ weeks & 465 \\
\hline Nationality & Albanian & 17 \\
\cline { 2 - 3 } & Foreign & 466 \\
\hline
\end{tabular}


Graphics 1-4 below show a schematic distribution of the 2017 data
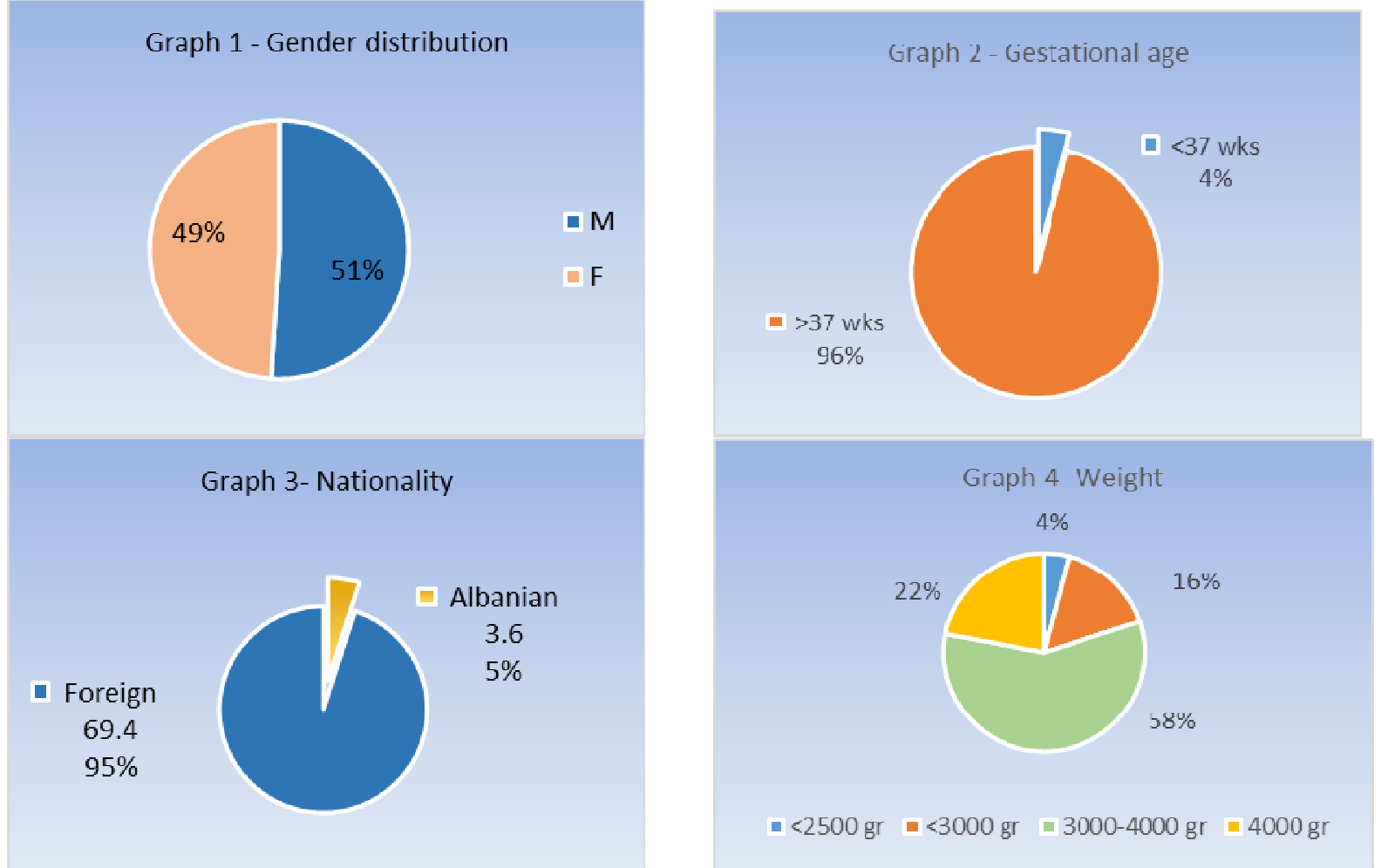

With respect to the 483 babies involved, 187 of them or $38.7 \%$ needed at least 1 blood test to evaluate VB levels. A total of 319 blood tests for VB were obtained from which it turns out that each baby was manipulated 1.7 times.

Cases of pathological hyperbilirubinemia that required treatment with phototherapy have been studied in particular. During the period March-September 2017, 28 babies or 5.7\% of those included in the study were treated with phototherapy, categorized respectively in the table 2 below.

Tab 2.

\begin{tabular}{|c|c|c|c|c|}
\hline & & Manipulated babies & Blood tests & Phototherapy \\
\hline Total & & 187 & 319 & 28 \\
\hline \multirow[t]{2}{*}{ Gender } & $\mathrm{M}$ & 90 & 148 & 11 \\
\hline & $\mathrm{F}$ & 97 & 171 & 17 \\
\hline \multirow[t]{4}{*}{ Weight } & $<2500$ gr & 14 & 29 & 1 \\
\hline & $<3000$ gr & 38 & 73 & 8 \\
\hline & $3000-4000 \mathrm{gr}$ & 134 & 199 & 17 \\
\hline & $>4000 \mathrm{gr}$ & 10 & 18 & 2 \\
\hline \multirow{2}{*}{$\begin{array}{l}\text { Gestational } \\
\text { age }\end{array}$} & $<37$ weeks & 14 & 32 & 3 \\
\hline & $>37$ weeks & 173 & 287 & 25 \\
\hline \multirow[t]{2}{*}{ Nationality } & Albanian & 180 & 306 & 27 \\
\hline & Foreign & 7 & 13 & 1 \\
\hline
\end{tabular}

In terms of cost-effectiveness, it was concluded that during this period for each newborn the cost is 0.7 of the VB value.

\section{Results from 2018.}

From March to August 2018, 539 babies were born at American Hospital 3, of which 531 babies were included in our study. 8 babies were excluded for the following reasons: 1 due to weight $<2000 \mathrm{gr}, 4$ for gestational age $<34$ weeks, 1 for associated pathologies for which they needed a long stay in NICU, 2 babies whose bilirubin was taken after the 14th day of life and 2 babies for incomplete data.

The following table (tab. 3) shows the data grouped by gender, weight, gestational age and nationality. 
Tab. 3

\begin{tabular}{|lll|}
\hline \multirow{2}{*}{ Total cases in the study } & $\mathbf{5 3 1}$ \\
& $\mathrm{M}$ & 272 \\
\cline { 2 - 3 } Weight & $\mathrm{F}$ & 259 \\
& $<2500 \mathrm{gr}$ & 16 \\
\cline { 2 - 3 } & $<3000 \mathrm{gr}$ & 95 \\
\cline { 2 - 3 } & $3000-4000 \mathrm{gr}$ & 381 \\
\cline { 2 - 3 } Gestational & $>4000 \mathrm{gr}$ & 39 \\
\hline age & $<37$ weeks & 28 \\
\hline Nationality & $>37$ weeks & 513 \\
& Albanian & 15 \\
\hline
\end{tabular}

Graphs 5-8 below show a schematic distribution of 2019 data.
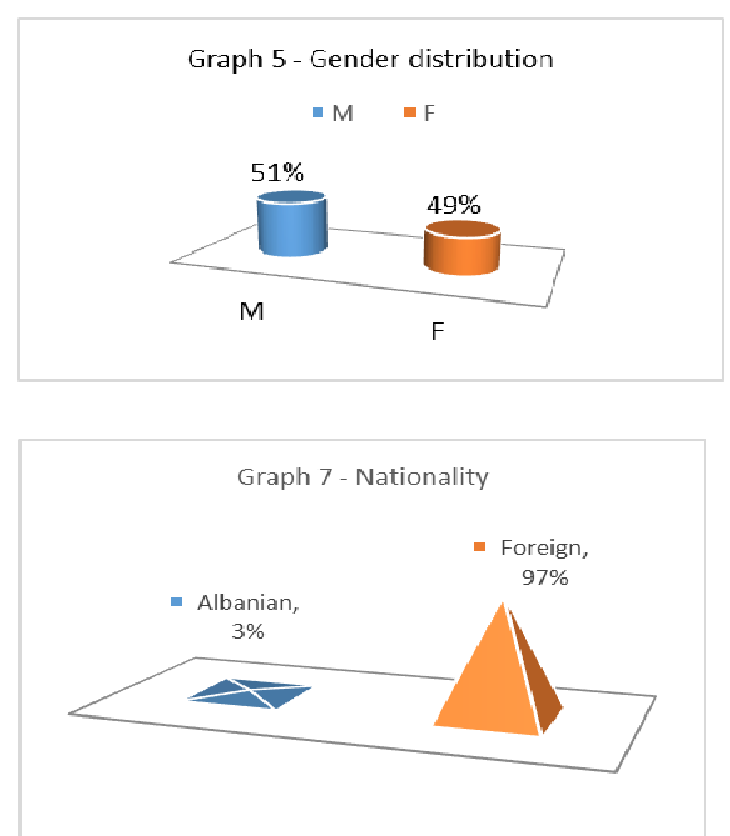

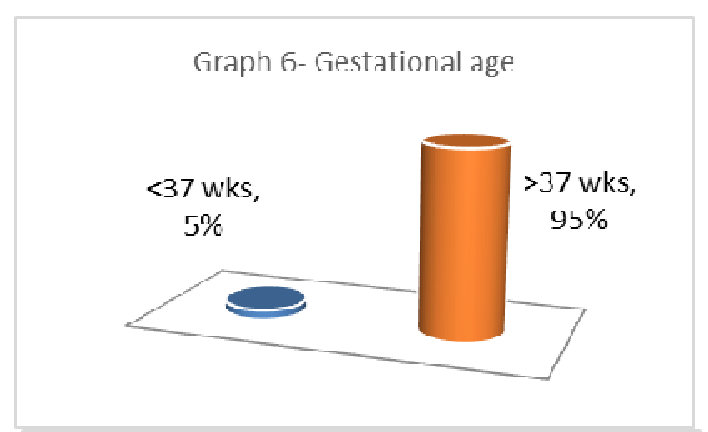

Graph 8 - Weight

- $<2500 \mathrm{gr} \quad \|<3000 \mathrm{gr}$

- $3000-4000 \mathrm{gr}=>4000 \mathrm{gr}$

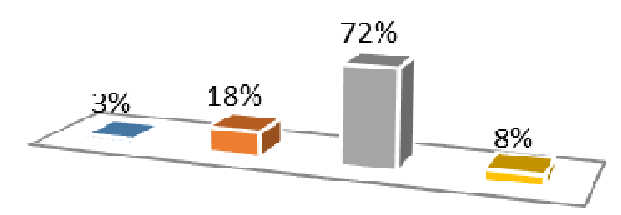

Out of 531 babies included in the study, 129 of them or $24.2 \%$ needed at least 1 blood test to evaluate BV levels. A total of 209 blood tests for BV were obtained from which it turns out that each baby was manipulated 1.61 times.

23 babies or $4.3 \%$ of them have pathological hyperbilirubinemia, who were treated with phototherapy.

The data found is shown in table 4 below:

Tab. 4

\begin{tabular}{|lllll|}
\hline \multirow{2}{*}{ Total } & & Manipulated babies & Blood tests & Phototherapy \\
\hline Gender & & 129 & 209 & 23 \\
\hline \multirow{2}{*}{ Weight } & $\mathrm{M}$ & 75 & 117 & 10 \\
\cline { 2 - 5 } & $\mathrm{F}$ & 54 & 92 & 13 \\
& $<2500 \mathrm{gr}$ & 12 & 19 & 1 \\
\cline { 2 - 5 } & $<3000 \mathrm{gr}$ & 33 & 61 & 9 \\
\hline Gestational & $3000-4000 \mathrm{gr}$ & 74 & 111 & 3 \\
\hline & $>4000 \mathrm{gr}$ & 10 & 43 & 7 \\
\hline Nage & $<37$ weeks & 18 & 166 & 16 \\
\cline { 2 - 5 } & $>37$ weeks & 111 & 202 & 22 \\
\hline
\end{tabular}

In terms of cost-effectiveness it was concluded that in this period for each newborn the cost is 0.39 of the 
VB value.

The following table (tab.5) shows the summarized results of the study.

Tab. 5

\begin{tabular}{|c|c|c|}
\hline & 2017 & 2018 \\
\hline No of newborns & 505 & 531 \\
\hline No babies in the study & 484 & 531 \\
\hline Manipulated babies & 193 or $39.8 \%$ & 129 or $24.2 \%$ \\
\hline Blood tests & 321 & 209 \\
\hline Blood tests per newborn & 1.66 & 1.61 \\
\hline Each baby cost & $\mathbf{0 . 6 6}$ (of $B V$ value) & 0.39 \\
\hline Phototherapy & 28 or $5.7 \%$ & 23 or $4.3 \%$ \\
\hline
\end{tabular}

\section{Discussions}

The bilirubin level is one of the most common reasons of blood tests in the neonatal period. The measurement of venous bilirubinemia is the gold standard, but considering that it is a painful examination that requires qualified staff and it is expensive, makes the use of TB a very important method to evaluate the neonatal jaundice ${ }^{9}$. Our study aimed to show that the use of TB measurement reduces the blood tests in babies, and consequently the costs. As many other studies, the measurement of TB although it has significant value in predicting bilirubin values it cannot replace VB.

The TB measurement is an appreciated method for monitoring neonatal jaundice, and it helps us for the right moment when to take the VB. ${ }^{7}$ The correlation between bilirubin levels in the skin and the central nervous system is still unclear, so the moment when the hemato-encephalic barrier is crossed remains unclear. The treatment of hyperbilirubinemia will remain one of the most common treatments in the neonatal period. The decision for phototherapy belongs always to the VB values . This is due to the fact that different TB measuring devices have different correlations levels between $\mathrm{TB}$ and $\mathrm{VB}$ 3,7,9.

In recent years use of technology in medicine, has improved tremendously the diagnosis and even treatment, as we can evaluate and monitor babies since intrauterine life by use of high definition medical devices like ultrasound. ${ }^{11,12}$ More we know about the health status of the baby in uterine life, more we are prepared to early diagnose and proper treatment in the neonatal life, using medical devices in medicine. ${ }^{11,13,14,15}$

So combining clinical medical knowledge with use of high tech in medicine, brings faster results even by being less invasive, like in transcutaneous bilirubin measurement by medical devices.

Besides the device, an important role is played by the skin color, melamine, subcutaneous tissue maturity, hemoglobin concentration ${ }^{5}$.

It should be mentioned that our hospital, being a private one, consists of a category of patients, both mothers and neonates, well followed during the study. All our patients have a very good perinatal care, we can know the risk factors better and interfere from the very beginning as well. All our newborns visit the doctor during their first week of life, which gives us the chance to test them in the right moment, but this also made our population limited for the study.

One of other limitations for the study was the VB measurement after the $2^{\text {nd }}$ week in some cases, which we chose to exclude, based on the physiological evaluation curves of bilirubin in the newborn.

With regard to the conclusions on the cost-effectiveness, it was based on relative data, on the basis of the VB cost of our laboratory, but certainly it is needed a more specific analysis, as it is very variable depending on the appliances available at each hospital. The conclusions will depend on the methods and laboratory equipment used and the type of transcutaneous bilirubin meter chosen by each one of them.

\section{Conclusion}

The close monitoring of newborns for the hyperbilirubinemia risk is part of all important guidelines. Sufficient evidence has arrived to recommend measuring TB as a non-invasive method. The use of TB to evaluate neonatal jaundice is one of the 10 most common researches in the field of neonatology. The use of TB measurement was used at first in 1980 taking an increasingly important role over the years. Monitoring neonatal jaundice through TB measurement is an increasingly popular method as it is fast, clean, saves time, does not require qualified staff, it can be repeated as many times as needs no extra cost, it is more cost effective than VB measurement and most importantly it is a non-invasive method, thus painless for newborns. The TB measurement is a very good method to evaluate neonatal jaundice, however, it does not replace in absolute mode the VB measurement. Recognizing its limitations, so far, the decision to treat hyperbilirubinemia is always based on VB values.

\section{References}

1. National perinatal clinical protocols, 2012 - Albania Ministry of Health 
2. E. Tushe et al. (2000). Use or misuse of technology.

3. Sankaran K. (2006). Transcutaneous bilirubinometry in neonates. Paediatrics \& child health, 11(2), 75-76. https://doi.org/10.1093/pch/11.2.75.

4. Watchko J. Recent advances in the management of neonatal jaundice. Research and Reports in Neonatology. 2014;4:183-193 https://doi.org/10.2147/RRN.S52373.

5. N. Bosschaart, J. H. Kok, A. M. Newsum, D. M. Ouweneel, R. Mentink, T. G. von Leeuwen, M. C. G. Aalders. Limitations and opportunities of transcutaneous bilirubin measurements, pediatrics vol.129. April 2012.

6. L. Briscoe, S. Clark, C W Yaxoll. Can transcutaneous bilirubinometry reduce the need for blood tests in jaundiced full term babies, bmj.com/content/86/3/F190.

7. B. van den Esker - Jonker, L. den Boer, R.M. C. Pepping, J. Bekhof. Transcutaneous bilirubinometry in jaundiced neonates: a randomized controlled trial, Pediatrics vol 138, December 2016.

8. Ch. Okwundu, O.A. Uthman, G.Suresh, J.Smith, Ch.S.Wiysonge, V K Bhutani. Transcutaneous bilirubinometry versus total serum bilirubin measurement for newborns, Cochrane database syst rev, may 2017.

9. L. Capasso, C. Parrella, A. C. Borrelli, R. Maffucci, P. Milite, A. Sodano, T. Ferrara, F. Raimondi. Is it worthwhile using a transcutaneous bilirubinometer in nursery?, Early Human Development- Elsevier 2012.

10. M. R. Rizvi, F. M. Alaskar, R. S. Albaradie, N. F. Rzvi, K. Al- Abdulwahab. A novel non invasive technique of measuring bilirubin levels using bilicapture, Oman Medical Journal, January 2019.

11. Celami, R. (2014). Obstetrical Ultrasound Examination And Biochemical Markers As Contemporary Tool Assessment For Fetal Anomalies In Albania. Advances in Life Science and Technology, 16, 45-48.

12. Basha, M., Celami, R., Preza, K., \& Kabili, G. (2015). Safety and Concerns of Diagnostic Imaging Utilisation during Pregnancy. Anglisticum Journal, 4.

13. Delija, Z., \& Celami, R. (2013). Current Point of View in Preterm Labor Management in Albania. Advances in Life Science and Technology, 12, 13-16.

14. Celami, R., Kalo, T., Klosi, H., Kabili, G., Milo, A.G., Horeshka, N., Marqeshi, A. (2015). Infections during Pregnancy; Accessibility, Consequences, Challenges and Appropriate Management Approach in Albania. Advances in Life Science and Technology, 37, 48-50.

15. Kabili, G., Celami, R., Daphnis, et al. (2017). Use of Biochemical Markers in Predictive and Diagnostic Means in Diagnosing Obstetrical Pathologies in Albania. Advances in Life Science and Technology, 52, 2426. 